\title{
Possible Association of Pulmonary Atresia with In-Utero Coxsackievirus B Exposure
}

\author{
Horacio G. Carvajal ${ }^{1}$ (1) $\cdot$ Vipul Sharma $^{1} \cdot$ Lisa S. Goessling ${ }^{1} \cdot$ Taylor C. Merritt $^{1} \cdot$ Anoop K. Brar $^{1} \cdot$ Pirooz Eghtesady $^{1,2}$
}

Received: 19 October 2021 / Accepted: 9 December 2021 / Published online: 12 January 2022

(c) The Author(s), under exclusive licence to Springer Science+Business Media, LLC, part of Springer Nature 2022

\begin{abstract}
Gestational viral infection has been associated with congenital heart disease (CHD). Few studies, however, have studied the potential role of gestational Coxsackievirus B (CVB) exposure in the pathogenesis of CHD. We prospectively enrolled women with pregnancies affected by CHD to explore possible associations with in utero CVB exposure. Serum samples were obtained from 122 women referred for fetal echocardiography between 2006 and 2018. We quantified CVB IgG and IgM levels, with titers $\geq 15.0 \mathrm{U} / \mathrm{mL}$ considered positive and measured neutralizing antibodies for three CVB serotypes: CVB1, CVB3, and CVB4. Using data from the national enterovirus surveillance system, we compared the annual exposure rates for each serotype in our cohort to infections reported across the United States. 98 pregnancies with no genetic defects were included. Overall, 29.6\% (29/98) had positive IgG and 4.1\% (4/98) of women had positive CVB IgM titers. To explore first-trimester CVB exposure, we focused exclusively on the 26 women with positive $\operatorname{IgG}$ and negative IgM titers. $61.5 \%$ (16/26) had neutralizing antibodies against a single serotype and 38.5\% (10/26) against multiple CVB serotypes. CVB4 neutralizing antibodies were the most common $(65.4 \%, 17 / 26)$, followed by CVB3 $(53.9 \%, 14 / 26)$ and CVB1 $(30.8 \%, 8 / 26)$. Among these, $30.8 \%$ of babies presented pulmonary valve anomalies: $19.2 \%(5 / 26)$ pulmonary atresia, and $11.5 \%(3 / 26)$ pulmonary stenosis. $23.1 \%$ (6/26) of babies had coronary sinusoids. CVB exposure in our cohort mirrored that of reported infections in the United States. Our results suggest a possible association between gestational CVB exposure and specific CHD, particularly pulmonary valve anomalies and coronary sinusoids.
\end{abstract}

\section{Introduction}

Maternal exposure to viruses during pregnancy has been associated with development of congenital heart defects (CHD), although the causative mechanisms remain unclear. Perhaps the best-known example is congenital rubella syndrome, where maternal infection can result in patent ductus arteriosus, pulmonary valve and artery anomalies, and ventricular septal defects (VSD) in the offspring [1]. Other pathogens, such as cytomegalovirus, have similarly been associated with CHD [2]. In women affected by febrile illness during pregnancy, the overall risk of the baby developing CHD increases twofold [3]. While a fever may develop

Pirooz Eghtesady

eghtesady670@wustl.edu

1 Section of Pediatric Cardiothoracic Surgery, Department of Surgery, Washington University in St. Louis School of Medicine, St. Louis, MO, USA

2 One Children's Place, Suite 6120, St. Louis, MO 63110, USA for many reasons, such as upper respiratory or urinary tract infections, the infectious agent responsible is often not identified $[4,5]$. Surprisingly, data regarding different infections during pregnancy and the outcome of the pregnancy are sparse. Botto et al. found febrile genitourinary infections were associated with selected heart defects, particularly right-sided obstructive defects $(\mathrm{OR}>3)$ and possibly others [4]. Similarly, Guo and colleagues found an increased risk of birth defects, including heart anomalies, in the setting of first trimester infections [6]. No study, to the best of our understanding, has specifically looked at prenatal gastrointestinal infections and neonatal outcomes.

Enteroviruses, part of the Picornaviridae family, are a common and varied group of viruses responsible for diseases such as polio, hand-foot-mouth disease, myocarditis, and viral meningitis [7]. The clinical presentation of these viruses can vary by age: neonates and children younger than five years typically present with myocarditis, aseptic meningitis, and encephalitis; children between 5 and 15 years old often suffer from central nervous system infections; and adults usually present with myocarditis [8]. In addition, 
many enteroviruses can result in asymptomatic infection [9]. Importantly, asymptomatic infection during pregnancy by common pathogens, such as enteroviruses, can result in subclinical viremia that can then lead to congenital anomalies [8]. Therefore, the incidence of viral infections by agents such as enteroviruses during pregnancy, along with the risk of vertical transmission to the fetus, may be underdiagnosed [10]. Epidemiologic studies done in the 1960s suggested an association between maternal enteroviral infection, particularly coxsackievirus B (CVB), and CHD [11]. Interestingly, the coxsackievirus and adenovirus receptor (CAR), through which CVB infects cardiomyocytes in myocarditis, is highly expressed in the developing heart and brain, playing a critical role in cardiac development during gestation [12-15].

We have previously shown that maternal CVB infection during early gestation can induce CHD, particularly VSD and abnormal myocardial architecture similar to ventricular non-compaction, in a murine model [16]. To investigate the potential clinical relevance of our animal studies, we conducted an exploratory study of women with pregnancies affected by CHD, analyzing serum samples for the presence of CVB-specific IgG and IgM antibody titers, as well as serotype-specific neutralizing antibodies and their relation to specific cardiac lesions.

\section{Methods}

\section{Patients}

Women over the age of 18 referred for fetal echocardiography during their second trimester of pregnancy due to concern for possible CHD between December 2006 and May 2018 were prospectively enrolled into the Washington University in St Louis Congenital Heart Registry after providing their informed consent. Those with twin or multiple gestations, a history of chromosomal anomalies, or treatment for infertility were ineligible for our study. Maternal serum was collected upon enrollment and used for antibody analysis. The women were followed to term, and their babies' echocardiographic reports were reviewed to confirm the presence and type of CHD diagnosis that was reported at enrollment. All babies diagnosed with CHD at birth are referred for genetics evaluation at our institution, if not already done prenatally. For the purposes of this study, babies with confirmed genetic defects on chromosomal microarray or karyotype analysis, and those with known syndromic associations (e.g., trisomy 21), VACTERL association, or heterotaxy were excluded from analysis. The study was approved by the Washington University School of Medicine Institutional Review Board.

\section{Coxsackievirus IgM and IgG Titer Assays}

Quantitative detection of Coxsackievirus IgM and IgG antibodies was performed with Serion ELISA classic kits ESR134M and ESR134G, respectively (QED Biosciences, Inc., San Diego, CA). The assays were performed as per kit instructions. Additionally, a positive control [IBO5041CON (IgM) or IBO5040CON (IgG), IBL America, Minneapolis, $\mathrm{MN}$ ] was run with each assay for test evaluation. Assays were carried out in duplicate. Antibody concentrations were calculated by inputting the assay absorbance readings and lot-specific curve parameters into the evaluation software supplied by the manufacturer. Antibody titers of $15 \mathrm{U} / \mathrm{mL}$ or greater were considered positive according to kit instructions and confirmed by comparison with positive controls.

\section{Assay of Neutralizing CVB Antibodies in Serum of Pregnant Women}

Neutralizing antibodies (NA) were measured against three CVB serotypes: CVB1-Chi07 (kindly provided by Dr. Xiaotian Zheng of Ann \& Robert H. Lurie Children's Hospital of Chicago), CVB3, Nancy, and CVB4, J.V.B. [American Type Culture Collection (ATCC) prototype strains]. Heatinactivated $\left(56{ }^{\circ} \mathrm{C}, 30 \mathrm{~min}\right)$ serum was initially diluted tenfold in culture media (Eagles Minimum Essential media supplemented with $2 \%$ fetal bovine serum and $2 \mathrm{mM}$ L-alanyl-L-glutamine) then further diluted two-fold in the same media. Serum dilutions were combined with $100 \mathrm{TCID}_{50}$ of virus and incubated at $37{ }^{\circ} \mathrm{C}$ for 90 min before being added to monolayers of BGMK-DAF cells (ATCC PTA-4594) in 96-well microtiter plates. Plates were incubated at $37{ }^{\circ} \mathrm{C}$ with $5 \% \mathrm{CO}_{2}$ and examined at day 5 post-infection for cytopathic effect (CPE). Results were expressed as the inverse final dilution that completely inhibited virus $\mathrm{CPE}$, with a titer of 80 or greater considered elevated.

\section{Statistical Analysis}

Data are expressed as median (interquartile range) or percent. Categorical values were compared using Pearson's Chi square test or Fisher's exact test, and Spearman's rho was used to test correlations. $p$ values less than 0.05 were considered statistically significant. Data are expressed as median (interquartile range) or percent. Since enteroviral infections can follow "mini-epidemic" patterns [17], we also analyzed CVB cases in our cohort to explore the seasonality of infections. To calculate the timing of first-trimester exposure, we subtracted the weeks of gestation at the time of enrollment from the enrollment date to determine the approximate time of conception. We then compared the annual incidence of 
first trimester CVB infections by serotype in our cohort to CVB1, CVB3, and CVB4 infections in the United States, as reported by the national enterovirus surveillance system (NESS) [18-20], as well as CVB infection data kindly provided by Dr. Allan Nix at the Centers for Disease Control and Prevention. Importantly, enterovirus reporting to the NESS relies on voluntary surveillance from participating labs and may thus not account for all enteroviral infections in the United States. Statistical analysis was conducted using SPSS version 25 (IBM Corporation, NY, USA).

\section{Results}

\section{Patients}

In total, 122 women were enrolled. After birth, 17 babies were diagnosed with genetic disorders, including Turner syndrome (1/17), Kabuki syndrome (1/17), Opitz syndrome (1/17), mandibulofacial dysostosis with microcephaly (1/17), and mutations of uncertain clinical significance (13/17). We additionally excluded two infants with VACTERL association and five with heterotaxy, resulting in 98 eligible subjects (Fig. 1). Demographic data for these women can be found in Table 1. Median age at enrollment was 28.1 (22.8-30.6) years, and median gestational age was 30 (28-33) weeks.

\section{Evidence of CVB Exposure/Infection in Pregnant Women}

Both IgG and IgM titers in serum samples collected at the time of enrollment were analyzed. Overall, $4.1 \%$ (4/98) of women had positive IgM titers and 29.6\% (29/98) had positive IgG titers, with three women presenting both. Based on our murine studies, where infection during early gestation resulted in the highest incidence of CHD [16], we hypothesized that CVB exposure, if relevant, had to have occurred early during the first trimester to be considered as possibly etiologic for CHD. Serological studies of patients with viral infections have shown that, in general, IgM levels start to decline 2 months after infection, and are usually negligible by the third month post-exposure [21]. Thus, we excluded
Table 1 Demographics for women included

\begin{tabular}{ll}
\hline & $N=98$ \\
\hline Patient age at enrollment (years) & $28.1(22.8-30.6)$ \\
Gestational age (weeks) & $30(28-33)$ \\
Race & \\
Caucasian & $64.8 \%(79 / 98)$ \\
African American & $15.3 \%(15 / 98)$ \\
Hispanic & $3.1 \%(3 / 98)$ \\
Asian & $1.0 \%(1 / 98)$ \\
Elevated IgM titers & $4.1 \%(4 / 98)$ \\
Elevated IgG titers & $29.6 \%(29 / 98)$ \\
\hline
\end{tabular}

the three subjects with both positive IgG and IgM titers, indicative of recent exposure during the second trimester, and focused solely on the remaining 26 women with positive IgG only. Among the 4 women excluded due to positive IgM titers, all babies were born with VSD along with different CHD: congenitally-corrected transposition of the great arteries (CC-TGA) $(50 \%, 2 / 4)$, pulmonary atresia (PA) $(25 \%$, $1 / 4$ ), and dextro-transposition of the great arteries (d-TGA) $(25 \%, 1 / 4)$.

The majority of these patients $(61.5 \%, 16 / 26)$ had elevated neutralizing antibodies against a single CVB serotype: 7.7\% (2/26) against CVB1, 19.2\% (5/26) against CVB3, and 34.6\% (9/26) against CVB4. In accordance with prior studies [9, 22, 23], we also observed a number of subjects with evidence of exposure to more than one CVB serotype. $3.8 \%(1 / 26)$ of women had elevated neutralizing antibodies against CVB1 and CVB4, 15.4\% (4/26) against CVB3 and CVB4, $7.7 \%(2 / 26)$ against CVB1 and CVB3, and 11.5\% (3/26) had elevated neutralizing antibodies against all three serotypes. CVB4 neutralizing antibodies were the most common among women with elevated titers against multiple serotypes.

\section{Association Between In-Utero CVB Exposure/ Infection and CHD}

Overall, the most common CHD were pulmonary stenosis $(18.4 \%, 18 / 98)$, double-outlet right ventricle (DORV)

Fig. 1 Subject selection algorithm

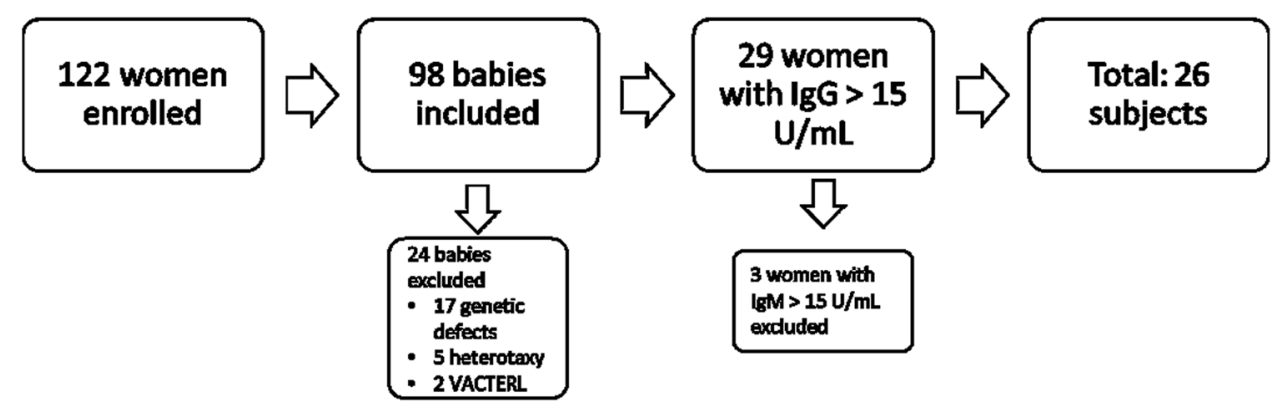


Table 2 Congenital heart defects, antibody titers, and characteristics of babies in the suspected first-trimester CVB exposure cohort

\begin{tabular}{|c|c|c|c|c|c|c|}
\hline \multirow[t]{2}{*}{ Primary diagnosis } & \multirow[t]{2}{*}{ Accompanying defects } & \multirow{2}{*}{$\begin{array}{l}\text { First } \\
\text { trimester } \\
\text { year }\end{array}$} & \multirow[t]{2}{*}{$\mathrm{IgG}(\mathrm{U} / \mathrm{mL})$} & \multicolumn{3}{|c|}{$\begin{array}{l}\text { NA titers for subjects with elevated } \\
\text { IgG }\end{array}$} \\
\hline & & & & CVB1 NA & CVB3 NA & CVB4 NA \\
\hline PA/IVS & Coronary sinusoids & 2014 & 144.1 & 10 & 640 & 10 \\
\hline HLHS & AA/MS & 2011 & 34.2 & 10 & 80 & 320 \\
\hline PA/IVS & Coronary sinusoids & 2013 & 33.9 & 10 & 80 & 160 \\
\hline Tetralogy of Fallot & PA & 2017 & 32.1 & 10 & 320 & 10 \\
\hline HLHS & AA/MS & 2013 & 30.7 & 10 & 10 & 160 \\
\hline d-TGA & & 2016 & 29.7 & 10 & 10 & 320 \\
\hline Tetralogy of Fallot & PA & 2008 & 29.3 & 320 & 20 & 640 \\
\hline HLHS & AA/MS, coronary sinusoids & 2013 & 29.3 & 10 & 20 & 80 \\
\hline Coarctation of the aorta & & 2016 & 28.4 & 10 & 10 & 80 \\
\hline HLHS & AA/MA, VSD & 2012 & 27.4 & 10 & 80 & 20 \\
\hline DORV HLHS variant & AS/MA & 2017 & 26.7 & 10 & 10 & 80 \\
\hline HLHS & AS/MS & 2010 & 26.6 & 160 & 160 & 640 \\
\hline Coarctation of the aorta & & 2015 & 26.5 & 10 & 10 & 80 \\
\hline DORV HLHS variant & AA/MA, thickened, stenotic pulmonary valve & 2017 & 25.8 & 320 & 640 & 20 \\
\hline DORV & PS & 2009 & 21.3 & 40 & 160 & 40 \\
\hline HLHS & AA/MS & 2008 & 19.7 & 160 & 640 & 320 \\
\hline PA/IVS & Tricuspid atresia & 2015 & 18.6 & 80 & 160 & 40 \\
\hline Tetralogy of Fallot & LCA to RV fistula & 2014 & 18.4 & 10 & 80 & 320 \\
\hline HLHS & AS/MS & 2011 & 17.7 & 10 & 10 & 80 \\
\hline HLHS & AS/MA, VSD & 2014 & 17.3 & 80 & 10 & 10 \\
\hline Coarctation of the aorta & VSD & 2007 & 17.2 & 10 & 20 & 640 \\
\hline HLHS & AS/MS, coronary sinusoids & 2015 & 16.8 & 80 & 80 & 80 \\
\hline d-TGA & & 2013 & 16.3 & 10 & 10 & 80 \\
\hline HLHS & $\begin{array}{l}\text { AA/MA, anomalous RCA from pulmonary } \\
\text { artery, RV trabeculations }\end{array}$ & 2012 & 16.3 & 20 & 160 & 640 \\
\hline Tetralogy of Fallot & PS & 2016 & 15.6 & 80 & 10 & 10 \\
\hline HLHS & AA/MS, coronary sinusoids & 2011 & 15.2 & 10 & 80 & 10 \\
\hline
\end{tabular}

$A A$ aortic atresia, $A S$ aortic stenosis, $D O R V$ double-outlet right ventricle, $d$-TGA dextro-transposition of the great arteries, $H L H S$ hypoplastic left heart syndrome, IVS intact ventricular septum, $L C A$ left coronary artery, $M A$ mitral atresia, $M S$ mitral stenosis, $P A$ pulmonary atresia, $P S$ pulmonary stenosis, $R C A$ right coronary artery, $R V$ right ventricle, $V S D$ ventricular septal defect

(16.3\%, 16/98), VSD (15.3\%, 15/98), and PA (14.3\%, 14/98). Within the CVB-exposed group, 30.8\% (8/26) of babies had pulmonary valve anomalies (Table 2). The most common of these was PA: three babies had PA with intact ventricular septum (PA/IVS), two with coronary sinusoids and one without, while two babies had tetralogy of Fallot (TOF) with PA. Three additional patients presented pulmonary valve anomalies: one had a thickened, stenotic pulmonary valve in the setting of DORV hypoplastic left heart syndrome (HLHS) aortic atresia/mitral atresia (AA/MA) variant, while two had pulmonary stenosis (one with DORV and another with TOF). Neutralizing antibodies against CVB3 were the most commonly found in this subset $(75 \%, 6 / 8)$; four women had isolated CVB3 neutralizing antibodies, one had antibodies against CVB3 and CVB4, and another against both CVB1 and CVB3.
Interestingly, 23.1\% (6/26) of babies with in-utero exposure to CVB also had coronary sinusoids. These were present in the two previously mentioned babies with PA/IVS, as well as three with HLHS. Of these, two had AA/mitral stenosis (MS), and one had aortic stenosis (AS)/MS subtype. Maternal serum samples found elevated neutralizing antibodies against a single CVB serotype in three instances (CVB3: 2; CVB4: 1), while the other two had neutralizing antibodies against various serotypes (CVB3 and 4: 1; CVB1, 3, and 4: 1). One patient with TOF had a fistula from the left coronary artery to the right ventricle, with elevated neutralizing antibodies against both CVB3 and CVB4. Babies with coronary sinusoids in the CVB-exposed subset comprised $50 \%(6 / 12)$ of all coronary sinusoid cases among the 98 enrolled women $(p=0.058)$, while $35.7 \%$ (5/9) of all babies with pulmonary atresia had CVB exposure $(p=0.295)$. 


\section{Seasonality of CVB Exposure}

To determine whether there were any patterns in CVB exposure in our cohort, we first calculated the estimated date of conception for women with elevated IgG and negative IgM levels. We then added the four women with elevated IgM, using the date of sample collection to the number of annual CVB cases, and compared the year of CVB exposure to the timing of study enrollment for the remainder of the cohort (Fig. 2). Notably, 67.3\% (66/98) of women were enrolled between 2012 and 2018, compared to 32.7\% (32/98) between 2006 and 2011. When controlling for the number of enrolled subjects, there was no correlation between CVB infection and year of exposure $\left(R^{2}=0.144, p=0.655\right)$.

Next, we were interested in comparing the specific CVB serotypes observed among the women with first-trimester exposure to the incidence of known CVB infections in the in $65.4 \%(17 / 26)$ of women with first-trimester exposure. Although there was an early peak of CVB4 infections in 2008, exposure rates remained high from 2010 onwards, with the greatest peak observed in 2013 (Fig. 3B). These data suggest that the risk of exposure among our cohort closely mirrors that of the general population in the United States.

\section{Discussion}

In this exploratory study, we analyzed the serum of 98 women referred for fetal echocardiography due to CHD during their second trimester of pregnancy. We found 26 women with evidence of possible CVB exposure in the first trimester, defined by elevated CVB-specific $\mathrm{IgG}$ without
Fig. 2 Annual incidence of CVB exposure over the study period

\section{CVB Infections over Study Period}

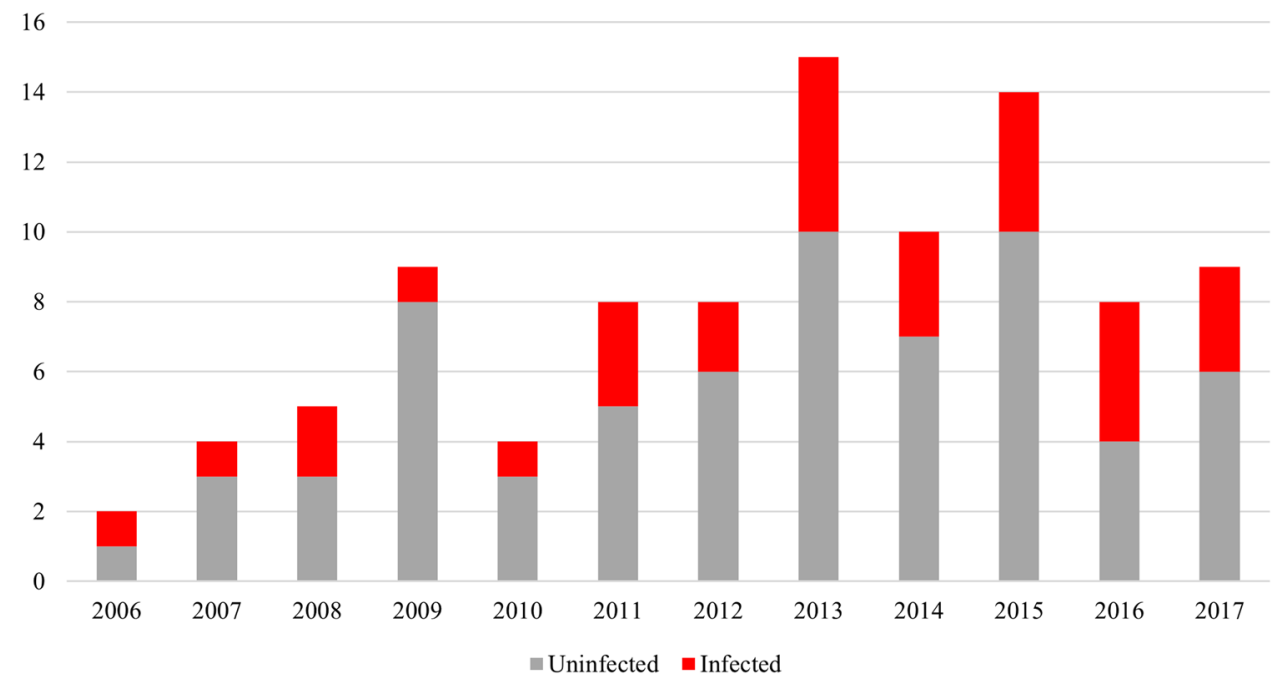

United States. CVB1 was the most common of the three CVB serotypes we studied reported by the NESS, comprising $36.5 \%$ (270/739) of infections. However, this was primarily driven by a significant infection peak in 2007 and 2008, with nearly negligible levels of CVB1 infection after 2009 (Fig. 3A) [18]. Although neutralizing antibodies against CVB1 were only present in $30.8 \%$ (8/26) of women with first-trimester exposure in our cohort, we also observed a peak in 2008 (Fig. 3B). CVB3 comprised 33.3\% (246/739) of reported cases, with important peaks observed in 2011 and 2014 in the United States. Similarly, CVB3 infections peaked in 2011 and 2014 in our cohort, with 53.9\% (14/26) presenting CVB3 neutralizing antibodies (Fig. 3B). Finally, CVB4 infections comprised 30.2\% (223/739) of those reported by the NESS, peaking in 2007 and 2013 (Fig. 3A). In our cohort, CVB4 was the most common serotype, found positive IgM titers at the time of sample collection. Among these, the most common CHD observed were pulmonary valve anomalies, particularly pulmonary atresia. Further, we noted an interesting association with the presence of coronary sinusoids, which was not strictly isolated to PA/IVS. Interestingly, although neutralizing antibodies against CVB4 were the most common overall, the majority of patients with pulmonary valve anomalies had elevated neutralizing antibodies against CVB3.

Viral infections during pregnancy have long been known to cause congenital defects. In the classic example of congenital rubella syndrome, the observation of seven babies with congenital cataracts and patent ductus arteriosus born to women infected with rubella during pregnancy in 1941 resulted in closer inspection of congenital defects in patients with similar gestational histories [24]. This eventually led 

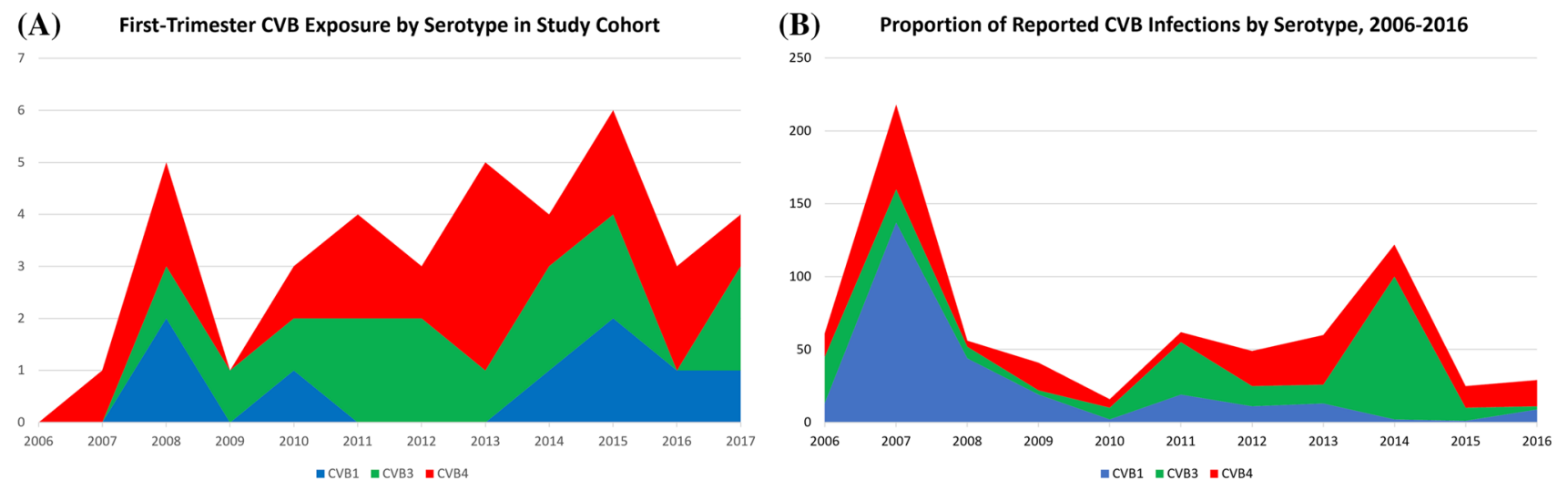

Fig. 3 A CVB infections observed in our cohort by serotype over the study period. Serotypes are not mutually exclusive. B CVB infections reported to the National Enterovirus Surveillance System in the United States between 2006 and 2016, by serotype

to the identification of rubella as the causative agent behind other congenital lesions, including pulmonary valve and artery anomalies, as well as congenital deafness, culminating in the creation of the rubella vaccine and subsequent vaccination campaign aimed at eradicating congenital rubella syndrome $[25,26]$. Similar studies focused on a possible association between gestational CVB infection and CHD were undertaken by Gordon et al. in the 1960s [11]. In a study analyzing the sera of 22,935 pregnant women, Gordon et al. found significantly higher rates of gestational CVB3 [10.1\% (14/139) vs. $2.7 \%$ (7/262)] and CVB4 [19.4\% (27/139) vs. $11.1 \%$ (29/261)] infection among mothers of babies with CHD compared to propensity-matched healthy controls. The most common CHD in this cohort included VSD and patent ductus arteriosus, followed by atrial septal defects, tricuspid, and aortic atresia. Although the study does not mention how CHD were diagnosed, these were likely established clinically or with B-mode echocardiography, given the technology available [27]. At the time, fetal echocardiography had yet to be developed [27], and echocardiographic assessment of the pulmonary valve would not be achieved until 1972 [28], limiting the study's results and possibly explaining the absence of pulmonary valve anomalies.

In our murine model of gestational CVB3 infection, the most prevalent CHD were VSD and abnormal myocardial architecture similar to ventricular non-compaction [16]. The abnormal myocardial architecture found in our murine model may be indicative of a pathological mechanism similar to the one observed in the formation of coronary sinusoids. In the case of coronary sinusoids, the coronary arteries form abnormal connections with the intertrabecular spaces of the ventricle, effectively connecting the epicardial coronary vessels with the ventricular lumen through defects in the myocardial architecture [29]. These defects have been mainly associated with PA/IVS and HLHS AA/ MS subtype, although they can also be present in other CHD
[30]. Coronary sinusoids have been hypothesized to arise from two distinct mechanisms. In the first, increased ventricular pressure in the setting of PA is purported to result in dilation of the intertrabecular spaces, and thus, abnormal ventriculocoronary connections [31]. Conversely, the second mechanism posits that coronary sinusoids are the primary defect and form independent of any ventricular outflow tract anomalies [31]. Although this second instance has been theorized to result from a second heart field defect, no causative agent has been identified [32]. Our murine study found that CVB infection suppressed cardiomyocyte proliferation, and we believed this accounted for both the presence of septal defects as well as abnormal myocardial architecture [16]. In light of our clinical findings, we are currently re-examining our murine samples for intramyocardial coronary vascular architecture, as these can be subtle or difficult to tease apart in the setting of altered trabeculation without specific staining for endothelial and coronary markers.

Pulmonary atresia, the second most common CHD among women with potential first-trimester CVB exposure in our cohort, can be an acquired lesion in babies with twin-twin transfusion syndrome [33]. Although the mechanism behind the observed lesion in these patients has not been clearly elucidated, it highlights the possible role of environmental factors in the pathogenesis of PA [34]. Notably, while older congenital rubella studies describe patent ductus arteriosus as the most common CHD, more recent studies have also found a high prevalence pulmonary valve and artery anomalies [35, 36]. Indeed, persistent patent ductus arteriosus may be an artifact of greater number of premature births, which can occur in the setting of congenital rubella. In countries where this disease has not been eradicated, congenital rubella remains one of the most important risk factors for right ventricular outflow tract obstruction [36]. These defects have been suggested to occur from the impairment of cardiac development, rather than direct myocardial damage 
$[37,38]$. An analysis of autopsy samples from babies with fatal congenital rubella syndrome found high expression of rubella antigens in myocardial and adventitial fibroblasts in samples taken from the heart, aorta, and pulmonary arteries, with no evidence of myocardial damage [39]. Given the results of our murine study, a similar mechanism, where CVB infection impairs the development of the fetal heart, may be responsible for the pulmonary valve anomalies observed in women with CVB exposures during early gestation. Moreover, the increased expression of CAR during fetal development [40] provides a plausible mechanism for CVB infection of the myocardium during a critical period of gestation, interfering with cardiogenesis and thus resulting in CHD.

Serologic testing is a well-established method of detecting viral exposure or prior infection, even in asymptomatic patients or those with mild symptoms [41]. Early studies of the role of viruses, such as rubella or cytomegalovirus infections, in the pathogenesis of congenital defects often relied on serologic testing [42, 43]. Indeed, a 1972 study found serological evidence of CVB infection in $36.7 \%(51 / 139)$ of women who gave birth to babies with CHD, compared to $26.7 \%$ (70/262) of healthy controls [22]. Interestingly, this study also reported that co-infection by more than one CVB serotype increased the teratogenic effect of gestational CVB infection. In our study, 38.5\% (10/26) of women with evidence of CVB infection had neutralizing antibodies for more than one serotype. Co-infection was most likely to occur with CVB3, with $90 \%$ (9/10) of women infected with multiple serotypes presenting neutralizing antibodies against CVB3. While coinfection with different enterovirus serotypes has been described, data regarding coinfection during pregnancy is scarce $[9,23,44]$. We did not observe any differences in CHD between women infected with a single or multiple CVB serotypes.

Finally, despite well-documented evidence of seasonal infection patterns among different enteroviruses [17, 45], we did not observe any pattern between CVB infection and observed CHD, likely due to our sample size. The prevalence of infections by specific serotypes observed in our cohort for the most part coincided with those reported by the NESS [18-20]. Notably, although CVB1 was the most common serotype reported in the United States over our study period, it was the least common serotype in our cohort. This was primarily due to an outbreak of severe neonatal CVB1 infection in the United States in 2007 and 2008, during which women experienced symptomatic third-trimester infections and the majority of infected neonates suffered from myocarditis [46]. We did not observe any CVB1 infections in 2007, possibly because only four patients were enrolled that year; however, both women with evidence of CVB exposure in 2008 had elevated neutralizing antibodies against CVB1.
Unfortunately, our sample size precludes us from drawing associations between specific CHD and CVB serotypes.

This study has several limitations. One of the most important is the small sample size, with only 98 subjects meeting the inclusion criteria. Of the 13 babies with genetic anomalies of unknown clinical significance, only one had potential first-trimester CVB exposure using our criteria. Our seasonality analysis was also limited by the small sample size. While the NESS provides the most comprehensive data regarding CVB infection in the United States, it relies on voluntary reports provided by participating laboratories. Thus, this data may be skewed towards more severe CVB infections mainly affecting newborns, complicating the assessment of seasonal trends for asymptomatic or minor infections that may nevertheless harm the developing embryo. Another important limitation was the heterogeneity of the CHD observed among enrolled babies, resulting in relatively small sample sizes for each specific defect. The goal of this study was to explore possible associations between specific cardiac defects and early gestational exposure to CVB, rather than to prove direct causation of CHD by CVB infection. Given our institution's status as a tertiary referral hospital, there was also a clear bias towards complex CHD. This is evidenced by the high prevalence of severe defects, such as HLHS, within our cohort. Unfortunately, fetal echocardiography is typically not performed until the second trimester of pregnancy following initial detection during routine obstetric ultrasound; consequently, another limitation of our study was the absence of samples obtained during the first trimester, which may have yielded important evidence of CVB infection during this critical period. To address these limitations, a larger prospective study of pregnant women with and without CHD-affected pregnancies is warranted. Ideally, this larger study would obtain samples during each trimester of pregnancy (with particular emphasis on the first trimester), comparing serologic data of those affected by CHD to those with healthy babies. Collection of stool samples, where enteroviruses can persist for several weeks, compared to only a few days in the blood, as well as serum samples at different points during pregnancy, with the aim of comparing the viral serotypes and concentration in the stool with serum antibody titers may further improve future studies [23, 47]. Prior studies have suggested possible associations between gestational CVB infection and neurological defects [10, 48-50], which is not surprising given the increased expression of CAR in the fetal central nervous system [13]. Although the focus of our study was solely on CHD, future studies may benefit from assessing the role of maternal CVB exposure on neurological defects.

Given the pervasiveness of enteroviral infections in the general population, their incidence during pregnancy may be vastly underestimated. Thus, the magnitude of their potential role in the etiology of CHD, especially in the 
case of simple defects that may not be diagnosed until after birth, is currently not known. Results from this exploratory study, as well as our murine model of gestational CVB infection, suggest that CVB infection during pregnancy may contribute to the burden of CHD in affected babies. Large prospective studies, with samples collected during the first trimester, are necessary to validate our findings. Confirmation of our findings in a larger cohort would provide a promising target for prevention of $\mathrm{CHD}$, mitigating the impact of CVB-associated CHD through public health measures, including screening, patient education, and potential vaccine development. Finally, additional analyses are needed to identify specific risk factors for increased susceptibility to these infections in women of childbearing age.

Supplementary Information The online version contains supplementary material available at https://doi.org/10.1007/s00246-021-02805-9.

Author Contributions HC reviewed and analyzed clinical and experimental data, drafted the manuscript. VS and LG contributed to experimental design, performed the antibody experiments and neutralization assays, collected experimental data. TM consented and enrolled subjects, collected samples. AB contributed to the experimental design and drafting of the manuscript. PE conceived and supervised the project, planned the experiments, and contributed to manuscript drafting. All authors contributed to critical revision of the manuscript.

Funding This project was partially supported by Award Number R01HL098634 from the National Heart, Lung, And Blood Institute. The content is solely the responsibility of the authors and does not necessarily represent the official views of the National Heart, Lung, And Blood Institute or the National Institutes of Health.

Data Availability This project used data from subjects enrolled in NCT03737006 and NCT01603732, found in www.clinicaltrials.gov. Data for patients who met the inclusion criteria can be found in the Supplemental Table.

Code Availability Not applicable.

\section{Declarations}

Conflict of interest The authors declare that they have no conflict of interest.

\section{References}

1. Jenkins KJ, Correa A, Feinstein JA et al (2007) Noninherited risk factors and congenital cardiovascular defects: current knowledge- a scientific statement from the American Heart Association Council on cardiovascular disease in the young. Circulation 115:2995-3014. https://doi.org/10.1161/CIRCULATIONAHA. 106.183216

2. Ye Z, Wang L, Yang T et al (2019) Maternal viral infection and risk of fetal congenital heart diseases: a meta-analysis of observational studies. J Am Heart Assoc 8:1-13. https://doi.org/ 10.1161/JAHA.118.011264

3. Botto LD, Erickson JD, Mulinare J et al (2002) Maternal fever, multivitamin use, and selected birth defects: evidence of interaction? Epidemiology 13:485-488. https://doi.org/10.1097/00001 648-200207000-00019

4. Botto LD, Panichello JD, Browne ML et al (2014) Congenital heart defects after maternal fever. Am J Obstet Gynecol 210:359.e1-359.e11. https://doi.org/10.1016/j.ajog.2013.10.880

5. Xia YQ, Zhao KN, Zhao AD et al (2019) Associations of maternal upper respiratory tract infection/influenza during early pregnancy with congenital heart disease in offspring: evidence from a case-control study and meta-analysis. BMC Cardiovasc Disord 19:1-13. https://doi.org/10.1186/s12872-019-1206-0

6. Guo L, Qu P, Zhang R et al (2018) Propensity score-matched analysis on the association between pregnancy infections and adverse birth outcomes in rural Northwestern China. Sci Rep. https://doi.org/10.1038/s41598-018-23306-5

7. Harvala H, Broberg E, Benschop K et al (2018) Recommendations for enterovirus diagnostics and characterisation within and beyond Europe. J Clin Virol 101:11-17. https://doi.org/10. 1016/j.jcv.2018.01.008

8. Muehlenbachs A, Bhatnagar J, Zaki SR (2015) Tissue tropism, pathology and pathogenesis of enterovirus infection. J Pathol 235:217-228. https://doi.org/10.1002/path.4438

9. Witsø E, Palacios G, Cinek O et al (2006) High prevalence of human enterovirus $\mathrm{A}$ infections in natural circulation of human enteroviruses. J Clin Microbiol 44:4095-4100. https://doi.org/ 10.1128/JCM.00653-06

10. Méreaux J, Picone O, Vauloup-Fellous C et al (2017) L'infection à entérovirus durant la grossesse: une cause sous-estimée de complications fœtale et néonatale ? Gynecol Obstet Fertil Senol 45:231-237. https://doi.org/10.1016/j.gofs.2017.02.004

11. Brown GC, Evans TN (1967) Serologic evidence of coxsackievirus etiology of congenital heart disease. JAMA 199:183. https:// doi.org/10.1001/jama.1967.03120030087016

12. Shi Y, Chen C, Lisewski U et al (2009) Cardiac deletion of the coxsackievirus-adenovirus receptor abolishes coxsackievirus B3 infection and prevents myocarditis in vivo. J Am Coll Cardiol 53:1219-1226. https://doi.org/10.1016/j.jacc.2008.10.064

13. Hotta Y, Honda T, Naito M, Kuwano R (2003) Developmental distribution of coxsackie virus and adenovirus receptor localized in the nervous system. Dev Brain Res 143:1-13. https:// doi.org/10.1016/S0165-3806(03)00035-X

14. Excoffon KJDA (2020) The coxsackievirus and adenovirus receptor: virological and biological beauty. FEBS Lett 594:1828-1837. https://doi.org/10.1002/1873-3468.13794

15. Sharma V, Perry DJ, Eghtesady P (2021) Role of coxsackieadenovirus receptor in cardiac development and pathogenesis of congenital heart disease. Birth Defects Res 113:535-545. https://doi.org/10.1002/bdr2.1860

16. Sharma V, Goessling LS, Brar AK et al (2021) Coxsackievirus B3 infection early in pregnancy induces congenital heart defects through suppression of fetal cardiomyocyte proliferation. J Am Heart Assoc. https://doi.org/10.1161/jaha.120.017995

17. Pons-Salort M, Grassly NC (2018) Serotype-specific immunity explains the incidence of diseases caused by human enteroviruses. Science 361:800-803. https://doi.org/10.1126/science. aat6777

18. Villarruel GR, Langley GE, Oberste MS, Pallansch M (2010) Nonpolio enterovirus and human parechovirus surveillanceUnited States, 2006-2008. Morb Mortal Wkly Rep 59:2006-2008

19. Abedi GR, Watson JT, Pham H et al (2015) Enterovirus and human parechovirus surveillance-United States, 2009-2013. Morb Mortal Wkly Rep 64:940-943. https://doi.org/10.15585/ mmwr.mm6434a3 
20. Abedi GR, Watson JT, Nix WA et al (2018) Enterovirus and parechovirus surveillance-United States, 2014-2016. Morb Mortal Wkly Rep 67:515-518. https://doi.org/10.15585/mmwr.mm671 $8 \mathrm{a} 2$

21. Mäkelä O, Lapinleimu K, Kostiainen E (1968) Timing of different virus neutralizing $19 \mathrm{~S}$ and $7 \mathrm{~S}$ antibodies in natural coxsackie infections of newborn babies and adults. Clin Exp Immunol 3:269-26976

22. Brown GC, Karunas RS (1972) Relationship of congenital anomalies and maternal infection with selected enteroviruses. Am J Epidemiol 95:207-217. https://doi.org/10.1093/oxfordjournals. aje.a121388

23. Stene LC, Rewers M (2012) Immunology in the clinic review series; focus on type 1 diabetes and viruses: the enterovirus link to type 1 diabetes: critical review of human studies. Clin Exp Immunol 168:12-23. https://doi.org/10.1111/j.1365-2249.2011. 04555.x

24. Gregg NM (1991) Congenital cataract following german measles in the mother. Epidemiol Infect 107:iii-xiv. https://doi.org/10. 5694/j.1326-5377.1941.tb50952.x

25. Mueller M (1969) Rubella vaccine is licensed. Science 165:48. https://doi.org/10.1126/science.165.3888.48

26. Papania MJ, Wallace GS, Rota PA et al (2014) Elimination of endemic measles, rubella, and congenital rubella syndrome from the Western hemisphere: the US experience. JAMA Pediatr 168:148-155. https://doi.org/10.1001/jamapediatrics.2013.4342

27. Maulik D, Nanda NC, Maulik D, Vilchez G (2017) A brief history of fetal echocardiography and its impact on the management of congenital heart disease. Echocardiography 34:1760-1767. https://doi.org/10.1111/echo.13713

28. Gramiak R, Nanda NC (1972) Echocardiographic detection of the pulmonary valve. Radiology 102:153-157. https://doi.org/10. 1148/102.1.153

29. Foker JE, Setty SP, Berry J et al (2008) Treatment of right ventricle to coronary artery connections in infants with pulmonary atresia and intact ventricular septum. J Thorac Cardiovasc Surg 136:749-756. https://doi.org/10.1016/j.jtcvs.2008.03.067

30. Jakamy R, Séguéla P-E, Valdeolmillos E et al (2019) Pulmonary atresia with ventriculocoronary arterial connections and a large conoventricular septal defect. JACC 1:545-548. https://doi.org/ 10.1016/j.jaccas.2019.09.008

31. Gittenberger-de Groot AC, Tennstedt C, Chaoui R et al (2001) Ventriculo coronary arterial communications (VCAC) and myocardial sinusoids in hearts with pulmonary atresia with intact ventricular septum: two different diseases. Prog Pediatr Cardiol 13:157-164. https://doi.org/10.1016/S1058-9813(01)00102-3

32. Gittenberger-de Groot AC, Jongbloed MRM, Wisse LJ, Poelmann RE (2010) Pulmonary atresia with intact ventricular septum: second heart field derived myocardial and epicardial developmental clues. Prog Pediatr Cardiol 29:3-9. https://doi.org/10.1016/j. ppedcard.2010.02.009

33. Lougheed J, Sinclair BG, Fung Kee Fung K et al (2001) Acquired right ventricular outflow tract obstruction in the recipient twin in twin-twin transfusion syndrome. J Am Coll Cardiol 38:15331538. https://doi.org/10.1016/S0735-1097(01)01549-2

34. Michelfelder E, Tan X, Cnota J et al (2015) Prevalence, spectrum, and outcome of right ventricular outflow tract abnormalities in twin-twin transfusion syndrome: a large, single-center experience. Congenit Heart Dis 10:209-218. https://doi.org/10.1111/ chd. 12215

35. Oster ME, Riehle-Colarusso T, Correa A (2009) An update on cardiovascular malformations in congenital rubella syndrome. Birth Defects Res Part A. https://doi.org/10.1002/bdra.20621

36. Ekure EN, Kalu N, Sokunbi OJ et al (2018) Clinical epidemiology of congenital heart disease in Nigerian children, 2012-2017. Birth Defects Res 110:1233-1240. https://doi.org/10.1002/bdr2.1361
37. Jackson BT (1968) The pathogenesis of congenital cardiovascular anomalies. N Engl J Med 279:80-89. https://doi.org/10.1056/ NEJM196807112790206

38. Naeye RL, Blanc W (1965) Pathogenesis of congenital rubella. JAMA 194:1277-1283. https://doi.org/10.1001/jama.1965.03090 250011002

39. Lazar M, Perelygina L, Martines R et al (2016) Immunolocalization and distribution of rubella antigen in fatal congenital rubella syndrome. EBioMedicine 3:86-92. https://doi.org/10.1016/j. ebiom.2015.11.050

40. Dorner AA, Wegmann F, Butz S et al (2005) Coxsackievirus-adenovirus receptor (CAR) is essential for early embryonic cardiac development. J Cell Sci 118:3509-3521. https://doi.org/10.1242/ jcs.02476

41. Yong SEF, Anderson DE, Wei WE et al (2020) Connecting clusters of COVID-19: an epidemiological and serological investigation. Lancet Infect Dis 20:809-815. https://doi.org/10.1016/ S1473-3099(20)30273-5

42. Alford CA, Schaefer J, Blankenship WJ et al (1967) A correlative immunologic, microbiologic and clinical approach to the diagnosis of acute and chronic infections in newborn infants. $\mathrm{N}$ Engl $\mathrm{J}$ Med 277:437-449. https://doi.org/10.1056/NEJM19670831277 0901

43. Weller TH, Alford CA, Neva FA (1964) Retrospective diagnosis by serologic means of congenitally acquired rubella infections. N Engl J Med 270:1039-1041. https://doi.org/10.1056/NEJM1 96405142702004

44. Kuramitsu M, Kuroiwa C, Yoshida H et al (2005) Non-polio enterovirus isolation among families in Ulaanbaatar and Tov province, Mongolia: prevalence, intrafamilial spread, and risk factors for infection. Epidemiol Infect 133:1131-1142. https://doi.org/10. 1017/S0950268805004139

45. Wikswo ME, Khetsuriani N, Fowlkes AL et al (2009) Increased activity of coxsackievirus B1 strains associated with severe disease among young infants in the United States, 2007-2008. Clin Infect Dis 49:2007-2008. https://doi.org/10.1086/605090

46. Centers for Disease Control and Prevention (CDC) (2008) Increased detections and severe neonatal disease associated with coxsackievirus B1 infection-United States, 2007. Morb Mortal Wkly Rep 57:553-556

47. Isaacs SR, Kim KW, Cheng JX et al (2018) Amplification and next generation sequencing of near full-length human enteroviruses for identification and characterisation from clinical samples. Sci Rep 8:1-9. https://doi.org/10.1038/s41598-018-30322-y

48. Dommergues M, Petitjean J, Aubry MC et al (1994) Fetal enteroviral infection with cerebral ventriculomegaly and cardiomyopathy. Fetal Diagn Ther 9:77-78. https://doi.org/10.1159/000263911

49. Euscher E, Davis J, Holzman I, Nuovo GJ (2001) Coxsackie virus infection of the placenta associated with neurodevelopmental delays in the newborn. Obstet Gynecol 98:1019-1026. https:// doi.org/10.1016/S0029-7844(01)01625-8

50. Gauntt CJ, Gudvangen RJ, Brans YW, Marlin AE (1985) Coxsackievirus group $\mathrm{B}$ antibodies in the ventricular fluid of infants with severe anatomic defects in the central nervous system. Pediatrics 76:64-68

Publisher's Note Springer Nature remains neutral with regard to jurisdictional claims in published maps and institutional affiliations. 